\title{
Autoimmune Hepatitis: 2019 Update
}

\author{
Atsushi Tanaka \\ Department of Medicine, Teikyo University School of Medicine, Tokyo, Japan
}

Autoimmune hepatitis (AlH) is a chronic inflammatory liver disease, characterized by the elevation of aminotransferases, presence of anti-nuclear antibody or anti-smooth muscle antibody, elevated immunoglobulin G (IgG), and interface hepatitis/plasma-lymphocytic inflammation based on histology. Recent epidemiological studies have indicated an increasing trend in the prevalence of $\mathrm{AlH}$ worldwide, especially in male patients; this trend may suggest the alteration of environmental triggers of disease onset over time. As no disease-specific biomarker or histological finding is currently available, AlH requires a clinical diagnosis, and a validated diagnostic scoring system with acceptable specificity and sensitivity has been proposed. Regarding treatment, corticosteroids and azathioprine are recommended, and in those who exhibit an incomplete response or those who are intolerant to these drugs, second-line therapy, such as mycophenolate mofetil, is considered. Overall, the long-term outcome is excellent in patients with complete biochemical responses, while life-long maintenance treatment may be required since the cessation of immunosuppressive agents frequently leads to the relapse of the disease. Acute-onset $\mathrm{AlH}$ does occur, and the diagnosis is very challenging due to the lack of serum autoantibodies or elevated IgG. The unmet needs include earlier diagnosis, intervention with disseminated clinical practice guidelines, and recognition and improvement of patients' health-related quality of life with the development of novel corticosteroid-free treatment regimens. (Gut Liver 2020;14:430-438)

Key Words: Immunosuppressive agents; Epidemiology; Environmental factors; Health-related quality of life; Clinical trial

\section{INTRODUCTION}

Autoimmune hepatitis (AIH) is a chronic liver disease of unknown etiology in which autoimmune-mediated relativities against hepatocytes are thought to play a crucial role. ${ }^{1}$ While middle-aged women have the highest risk for developing $\mathrm{AIH}$, patients in childhood or adolescence are not uncommon. The elevation of transaminases, detectable autoantibodies, such as anti-nuclear antibodies (ANA) or anti-smooth muscle antibodies (SMA), elevation of serum immunoglobulin G (IgG) levels, and interface hepatitis or plasma cell infiltration in liver histology are characteristic features of $\mathrm{AIH}^{2}{ }^{2}$ However, since there is currently no specific biomarker for the diagnosis of $\mathrm{AIH}$, criteria consisting of several items are used for diagnosis. ${ }^{3,4} \mathrm{AIH}$ responds very well to immunosuppressive drugs, and corticosteroids are the first-line treatment. ${ }^{5,6}$ While the overall survival of patients with AIH is comparable to that of those in the general population when the treatment response is favorable, the outcome is poor in patients with more than two relapses, even with corticosteroid treatment during the clinical course. ${ }^{7}$ In this review, we summarize recent studies in terms of the epidemiology, etiology, diagnosis, and treatment of AIH, and finally discuss the unmet needs in $\mathrm{AIH}$ as a future perspective.

\section{EPIDEMIOLOGY: AN INCREASING TREND}

AIH commonly affects middle-aged women, but occurrence in childhood or adolescence is observed. ${ }^{8,9}$ Worldwide, the peak incidence of AIH is around 50 to 60 years of age, and the highest incidence was observed around 60 to 70 years in both South Korea and Japan. ${ }^{9,10}$ Similarly to other autoimmune diseases, female preponderance is distinct in $\mathrm{AIH}$, and the male-to-female ratio is around $1: 4$ to $1: 6$.

The burden of AIH appears to be increasing worldwide, and the point prevalence reported in the published literature after

Correspondence to: Atsushi Tanaka

Department of Medicine, Teikyo University School of Medicine, 2-11-1, Kaga, Itabashi-ku, Tokyo 173-8605, Japan

Tel: +81-3-3964-1211, Fax: +81-3-3964-6627, E-mail: a-tanaka@med.teikyo-u.ac.jp

Received on July 24, 2019. Revised on August 30, 2019. Accepted on September 5, 2019. Published online April $20,2020$.

pISSN 1976-2283 eISSN 2005-1212 https://doi.org/10.5009/gnl19261

(c) This is an Open Access article distributed under the terms of the Creative Commons Attribution Non-Commercial License (http://creativecommons.org/licenses/by-nc/4.0) which permits unrestricted non-commercial use, distribution, and reproduction in any medium, provided the original work is properly cited. 
2000 is shown in Fig. 1.,11-18 The point prevalence of AIH is reported to be 10 to 25 per 100,000 population in European countries, and 5 to 25 in the Asia-Pacific region. Among these, epidemiological studies were serially conducted twice in Sweden and Japan. ${ }^{11,18,19}$ Although the study design, study area, and case findings were different, the point prevalence in Sweden was 10.7 and 17.3 in 2003 and 2009, respectively, indicating a 1.7fold increase in 6 years. ${ }^{11,18}$ Likewise, in Japan, the prevalence of AIH was 8.7 and 23.9 in 2004 and 2016, respectively, and thus tripled over a 12 -year period. ${ }^{19}$ It is also of note that although $\mathrm{AIH}$ was thought to be less frequent in Asia, the point prevalence recently reported is similar to that of the rest of the world (Fig. 1).

The male-to-female ratio of the population with $\mathrm{AIH}$ is considered to have changed over time, indicating a relative increase in the number of male patients. In Japan, the male-to-female ratio was 1:7 in 2004 and 1:4 in 2016. ${ }^{19}$ Similarly, other recent epidemiological studies of AIH indicate that the male-to-female ratio is approximately $1: 4$ to $1: 6$ for $\mathrm{AIH},{ }^{8,13,17,20}$ which is significantly higher than that reported previously (approximately 1:9 to $1: 10)$.

\section{ETIOLOGY: PERSPECTIVE FROM THE INTERACTION OF GENETICS AND ENVIRONMENT}

$\mathrm{AIH}$ is multifactorial and involves the interaction of both genetic background and environmental triggers; accordingly, environmental insult in genetically susceptible individuals may result in aberrant immunological reactions and lead to autoimmune-mediated injury to hepatocytes. Familial clustering of AIH is frequently reported, even though the risk of AIH in the family is relatively low; ${ }^{21}$ this indicates the importance of both genetic determinants and environmental triggers. To date, numerous susceptible loci for AIH in the human leucocyte antigen (HLA) and non-HLA region have been identified, and a genome-wide

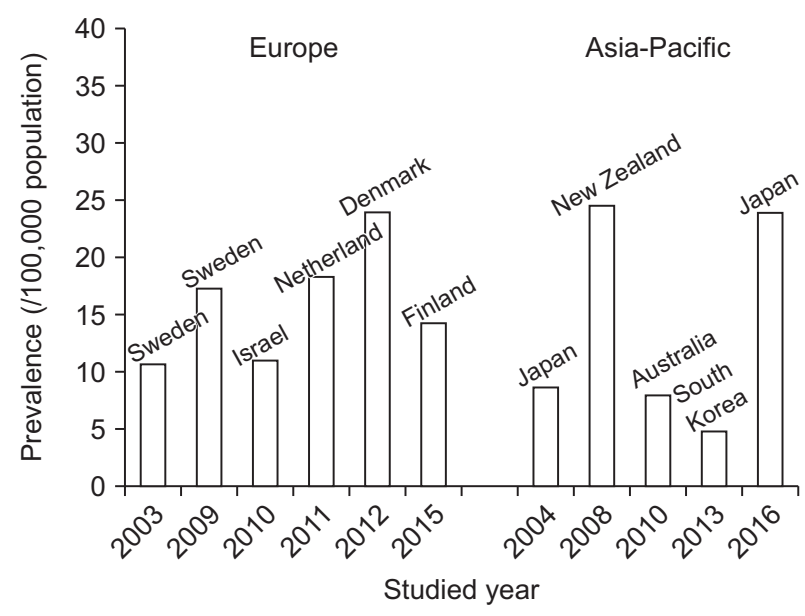

Fig. 1. The point prevalence (per 100,000 population) of autoimmune hepatitis in Europe and the Asia-Pacific region, reported after 2000. association study (GWAS) in the Netherlands determined HLA$D R B 1^{*} 0301$ and $D R B 1^{*} 0401$ to be the most relevant susceptible genotypes for $\mathrm{AIH}$, while $S H 2 B 3$ and CARD10 (genes in the non-HLA region) were shown to be significantly associated with $\mathrm{AIH} .^{22}$ Nevertheless, the associations of non-HLA loci have not yet been confirmed by other GWAS, indicating a strong contribution of HLA loci to disease susceptibility. Furthermore, recent epidemiological studies have suggested a relative importance of environmental triggers. ${ }^{19}$ As described, an increased trend of the prevalence of $\mathrm{AIH}$, especially in males, has been observed worldwide. This might be explained by a better awareness and recognition of $\mathrm{AIH}$ among physicians, while novel environmental factors contributing to the susceptibility of AIH and shared by both Europe and Asia-Pacific areas, and both sexes, might play a more crucial role in AIH. A well-designed case-control study is warranted to seek and identify environmental triggers in lifestyle, food, and beverages, or in chemicals, antibiotics, and xenobiotics, which trigger innate and adaptive immune responses leading to a breakdown of tolerance against hepatocytes. ${ }^{19}$

\section{DIAGNOSIS: A CLINICAL DIAGNOSIS}

\section{Presentation}

In typical cases, the presentation of $\mathrm{AIH}$ is insidious and patients with decompensated symptoms are rather rare, while the majority of patients with AIH have an acute-onset with general fatigue or jaundice, as described below. The elevation of transaminases, detectable autoantibodies (ANA or SMA), and elevated serum IgG level are common features captured by blood testing. Besides ANA or SMA, liver kidney/microsome (LKM)1 antibody or soluble liver antigen is occasionally detectable in $\mathrm{AIH}$, and the presence of LKM-1 characterizes type $2 \mathrm{AIH}$, while detectable ANA or SMA is a feature of type $1 \mathrm{AIH}$. A liver biopsy is required for the diagnosis of $\mathrm{AIH}$, and typical findings

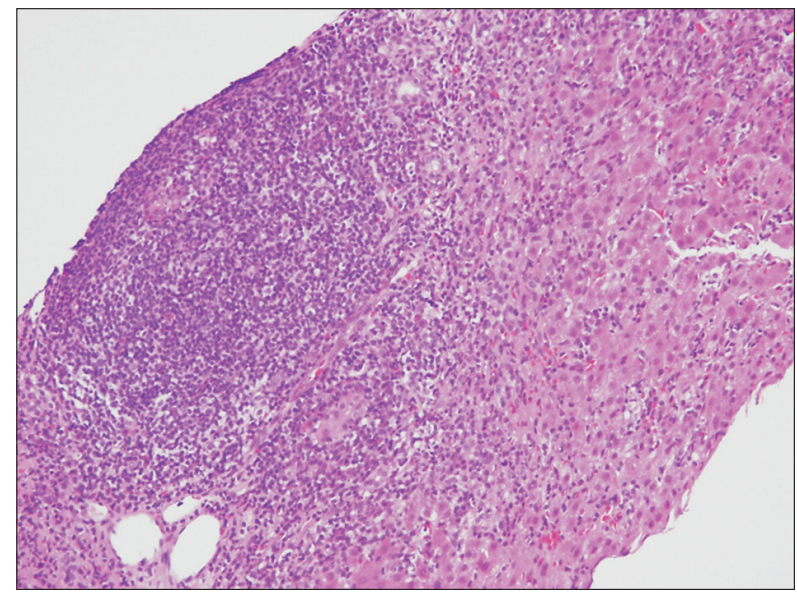

Fig. 2. Lymphocytic/lymphoplasmocytic infiltrates in the portal area and interface hepatitis; typical histological findings of autoimmune hepatitis $(\mathrm{H} \& \mathrm{E}, \times 100)$. 
of histology include interface hepatitis and lymphocytic/lymphoplasmocytic infiltrates (Fig. 2), in addition to emperipolesis, and hepatic rosette formation. However, the detection of ANA and SMA is not specific for AIH, and these histological findings are occasionally observed in other etiologies of hepatitis. Indeed, Gurung et al. ${ }^{23}$ recently demonstrated that while Kupffer cell hyaline granules, prominence of plasma cells in portal tracts, and plasma-lymphocytic inflammation are significantly associated with AIH, rosette formation and emperipolesis lacked significance when compared to chronic hepatitis of other etiologies. Thus, no biomarkers with high sensitivity and specificity (such as anti-mitochondrial antibodies in primary biliary cholangitis) are available for $\mathrm{AIH}$, and a combination of clinical parameters as diagnostic criteria has been used for the diagnosis of AIH.

Among a number of histological findings of AIH, liver fibrosis staging is crucial for predicting long-term outcome. Although close monitoring of fibrosis staging is essential during the clinical course, it is not feasible to carry out serial liver biopsies, and the role of noninvasive indices for fibrosis staging has been paid more attention to recently. These indices include biochemical calculation scores, such as the aspartate aminotransferase/platelet ratio (APRI) or fibrosis index based on the 4 factors (FIB-4), ${ }^{24}$ Mac-2 binding protein glycosylation isomer, ${ }^{25}$ magnetic resonance elastography, ${ }^{26}$ and transient elastography. ${ }^{27,28} \mathrm{~A}$ recent systematic review indicated that transient elastography had a good performance for determining significant fibrosis, advanced fibrosis, and cirrhosis, whereas APRI and FIB-4 showed poor performance. $^{29}$

\section{Diagnostic criteria}

The diagnostic criteria for AIH were created as a diagnostic scoring system by the International AIH Group in $1993,^{30}$ and revised in 1999. ${ }^{3}$ This scoring system was developed for the purification of patients with $\mathrm{AIH}$ for clinical research and trials, and was considered too complicated for daily use. In addition, atypical AIH patients (anti-mitochondrial antibody positive $\mathrm{AIH}$ or anti-hepatitis $\mathrm{C}$ virus antibody positive $\mathrm{AIH}$ ), who are required to be treated as typical AIH cases in daily practice, tend to be excluded by the deduction of points. Thereafter, a simplified scoring system to ease clinical application was proposed in $2008 ;^{4}$ this system consisted of only four items (Table 1): Autoantibodies (ANA or SMA), IgG, liver histology, and the absence of viral hepatitis. This is user-friendly and easy to determine whether corticosteroid treatment should be commenced or not. Even though retrospective, high specificity and specificity of the simplified scoring system in the diagnosis of AIH has been validated. ${ }^{31,32}$ A recent systematic review suggested that the simplified scoring system also provided high specificity and moderate sensitivity for the diagnosis of pediatric $\mathrm{AIH}^{33}$ On the other hand, in a Korean cohort of patients with AIH $(n=343$; mean age, 52.8 years old), definite/probable $\mathrm{AIH}$ according to the revised and simplified criteria were found to be $24.8 \% / 65.4 \%$ and
Table 1. Simplified Diagnostic Criteria for AIH

\begin{tabular}{llc}
\hline \multicolumn{1}{c}{ Variable } & \multicolumn{1}{c}{ Cutoff } & Point \\
\hline Autoantibodies & ANA or SMA 1:40 & 1 \\
& ANA or SMA $\geq 1: 80$ & 2 \\
& LKM $(\geq 1: 40)$ or SLA positive & 2 \\
IgG & $>$ ULN & 1 \\
& $>1.1 \times$ ULN & 2 \\
Liver histology & Compatible & 1 \\
& Typical & 2 \\
Absence of viral hepatitis & Yes & 2 \\
\hline
\end{tabular}

$\geq 6$, Probable autoimmune hepatitis (AIH); $\geq 7$, definite AIH.

ANA, anti-nuclear antibodies; SMA, anti-smooth muscle antibodies; LKM, liver kidney/microsome; SLA, soluble liver antigen; IgG, immunoglobulin G; ULN, upper limit of normal.

$34.4 \% / 38.5 \%$, respectively, and the concordance rate was quite low (38.8\%), with modest sensitivity of the simplified criteria. ${ }^{10}$

Regarding the diagnosis of $\mathrm{AIH}$, making a diagnosis of $\mathrm{AIH}$ after progression to a cirrhotic stage is common in both developed and developing countries. In a cohort in Pakistan and India, $84 \%$ and $71 \%$ of patients had evidence of clinical cirrhosis, respectively, with evident symptoms such as jaundice or abdominal distension. ${ }^{34}$ Moreover, a Korean study indicated that $22.7 \%$ of patients were cirrhotic at the stage of diagnosis. ${ }^{10}$ Even in a multicenter study in the United Kingdom, 21\% of patients had clinical decompensation and/or a MELD (Model for End-Stage Liver Disease) score >15 at presentation, and delayed diagnosis (time from first abnormal liver tests to diagnosis was $\geq 1$ year) was observed in $19 \%$ of patients. ${ }^{35}$ It is extremely important to have a suspicion of $\mathrm{AIH}$ in cases with elevated transaminases and make a diagnosis of $\mathrm{AIH}$ at an earlier fibrotic stage, prior to progression to cirrhosis.

\section{TREATMENT: CORTICOSTEROIDS AND BEYOND}

\section{Goal and indication of treatment for AlH}

The aim of treatment in AIH is to achieve complete biochemical and histological remission, and prevent further progression of liver fibrosis. Complete biochemical remission is associated with the normalization of both serum alanine aminotransferase (ALT) and IgG levels. Whereas increased mortality is observed in AIH patients with cirrhosis compared to those without cirrhosis, ${ }^{36}$ histological regression of fibrosis, as well as an improved long-term outcome will be attained by complete biochemical remission. ${ }^{37,38}$ Although the patients with AIH who should be treated are poorly defined, those with advanced or active AIH (advanced fibrosis, cirrhosis, ALT $\geq 3$ xupper limit of normal [ULN]) are expected to have a poor outcome and absolute indication for treatment. On the other hand, patients with mild disease (ALT <3 $\times$ ULN and mild or no fibrosis on histology) may not require prompt treatment, and be closely monitored until 
elevation of ALT and/or IgG. ${ }^{5}$

\section{First-line medical treatment}

Immunosuppressive treatment, consisting of either corticosteroids or a combination of corticosteroids and azathioprine, has been shown to improve the long-term outcome of patients with $\mathrm{AIH},{ }^{39}$ and is consistently recommended as a first-line treatment. ${ }^{5,40}$ While the American Association for the Study of the Liver Disease guidelines recommend either prednisone monotherapy (60 mg/day) or a combination of prednisone (30 $\mathrm{mg} /$ day) and azathioprine (50 mg/day), ${ }^{40}$ the European Association for the Study of the Liver (EASL) guidelines suggest 0.5-1 $\mathrm{mg} / \mathrm{kg} /$ day predniso(lo)ne as initial treatment, followed by a $50 \mathrm{mg} /$ day azathioprine add-on. ${ }^{5}$ Indeed, the recommended starting dose of predniso(lo)ne, $0.5-1 \mathrm{mg} / \mathrm{kg} /$ day, is somewhat ambiguous and leaves room for variation. Recently, Pape et al. ${ }^{41}$ retrospectively investigated whether a higher $(\geq 0.5 \mathrm{mg} / \mathrm{kg} /$ day) or lower ( $<0.5 \mathrm{mg} / \mathrm{kg} /$ day) initial dose of predniso(lo)ne affected the rate of the normalization of transaminases, and found no significant difference between the two groups, even after adjusting clinical confounders; they concluded that the dose of predniso(lo)ne is less relevant than previously assumed.

To avoid various adverse effects of predniso(lo)ne, budesonide has been suggested as an alternative to predniso(lo)ne. Budesonide has a 90\% first pass hepatic clearance rate and has less pronounced adverse effect profiles. In a prospective, doubleblind, randomized trial, treatment with budesonide in combination with azathioprine for non-cirrhotic AIH patients induced biochemical remission more effectively than prednisone with azathioprine, and had a significantly lower incidence of steroidspecific adverse effects. ${ }^{42}$ However, a case report appearing immediately after this trial, demonstrated that reactivation of $\mathrm{AIH}$ occurred during budesonide monotherapy and subsided with prednisone treatment. ${ }^{43}$ In a recent retrospective study in Germany, a biochemical response was observed in 70\% of patients after 12 months of treatment with budesonide monotherapy switched from prednisolone, and in 67\% of patients after 24 months. At the last follow-up evaluation (63 months on average), 25\% of patients received prednisolone therapy because of an insufficient response to budesonide or its side effects. ${ }^{44}$

\section{Second-line medical treatment}

In a questionnaire-based search among experts, mycophenolate mofetil (MMF) was the most frequently used a second-line treatment for AIH in the real world. ${ }^{45} \mathrm{MMF}$ is an ester prodrug of mycophenolic acid and is indicated for immunosuppression following organ transplant or lupus nephritis. MMF inhibits inosine monophosphate dehydrogenase, leading to the depletion of guanine nucleotides and ultimately the inhibition of DNA synthesis. ${ }^{46}$ Although not officially approved for AIH, MMF has been shown to be effective and well tolerated in several retrospective studies of AIH patients who responded insufficiently, or were intolerant, to corticosteroids. For instance, Efe et al. ${ }^{47}$ retrospectively collected data from 121 patients who received MMF as a second-line therapy, and found a complete response rate in 91.9\% and 34\% in corticosteroid-intolerant and insufficient responders, respectively. Recent systematic reviews demonstrated the overall efficacy of MMF as second-line therapy in $\mathrm{AIH}$, with a low discontinuation rate due to side effects. ${ }^{48,49}$ Other second-line treatments used by experts include tacrolimus and cyclosporine, and sirolimus, infliximab, and rituximab. ${ }^{45}$

Since B-cells play a significant role in the pathogenesis of $\mathrm{AIH}, \mathrm{B}$ cell depletion is a promising strategy for the management of AIH. Ianalumab (VAY736) is a B cell activating factor receptor-blocking, monoclonal antibody that works as a B celldepleting agent, and a clinical trial for primary Sjögren syndrome revealed therapeutic benefits without major side effects. ${ }^{50}$ Currently, a global, prospective, randomized phase 2/3 trial is being conducted for patients with $\mathrm{AIH}$ who exhibited an insufficient response, or were intolerant, to corticosteroids (ClinicalTrials.gov. NCT03217422).

\section{Withdrawal of immunosuppressive agents}

It is well known that the long-term administration of predniso(lo)ne frequently accompanies a variety of adverse effects, including osteoporosis, exacerbation of diabetes, cataracts and glaucoma, psychosis, cosmetic changes, and malignancy. Azathioprine, considered to be less toxic than corticosteroids, has a potential to lead to the development of leukopenia and thrombocytopenia, and in some cases, malignancy. In this regard, cessation of immunosuppressive drugs should be considered after achieving clinical remission. In line with this, remission of AIH should be strictly defined and maintained for an extended period. Remission of AIH is defined biochemically and histologically; biochemical remission consists of normalization of serum transaminases and IgG, and histological remission involves the disappearance of interface hepatitis, usually achieved long after serological remission. Even if both serum transaminases and IgG are normalized, residual interface hepatitis is often still present, resulting in relapse of AIH after cessation of treatment. Therefore, clinical practice guidelines recommend that treatment should be continued for at least 3 years, and for at least 2 years after complete normalization of serum transaminases and IgG levels. ${ }^{5}$ Thereafter, termination of therapy can be considered, but close observation with frequent testing of liver enzymes is required since relapse frequently occurs after withdrawal of immunosuppressive treatment (50\% to 90\%) and is typically observed in the first 12 months after withdrawal. When relapse of $\mathrm{AIH}$ occurs, treatment regimens correspond to initial treatment protocols with predniso(lo)ne and/or azathioprine, and are equally effective. However, it kept in mind that patients who relapse more than twice have a significantly worse outcome than those without relapse. ${ }^{7}$ In this regard, life-long treatment with maintenance doses of immunosuppressive drugs 
(predniso(lo)ne $5 \mathrm{mg} /$ day and/or azathioprine 50-100 mg/day) is an alternative option to avoid disease relapse, with intensive care for side effects.

\section{Liver transplantation}

AIH patients with end-stage liver disease or acute liver failure who are unable to be saved with current medical treatment require liver transplantation (LT). Although data regarding LT in patients with $\mathrm{AIH}$ are limited, the overall survival rate in $\mathrm{AIH}$ appears to be excellent in AIH: The 5- and 10-year recipient survival rates are $76 \%$ to $79 \%$ and $67 \%$ to $75 \%$, respectively, which are better than for most other indications for LT (Table 2). ${ }^{51-53}$ On the other hand, recurrence of $\mathrm{AIH}$ in the graft after LT is common, and it is very challenging to determine the incidence of recurrent $\mathrm{AIH}$, which is reported to be in the range of 7\% to $42 \%$ (Table 2). ${ }^{54-64}$ The inconsistency among studies is likely due to differences in diagnostic criteria, histological analysis (protocol or event-driven biopsy), small sample size in each study (no study with more than 100 patients enrolled), and follow-up time. ${ }^{65,66}$ The rate of recurrence increases as the follow-up time increases after LT. ${ }^{55,61,62}$ Neither the revised criteria, ${ }^{3}$ nor the simplified criteria, ${ }^{4}$ are validated for the diagnosis of recurrent $\mathrm{AIH}$.

A number of factors are reported to be associated with the re- currence of $\mathrm{AIH}$, including the severity of pre-transplant $\mathrm{AIH}^{54,61}$ and withdrawal of corticosteroids. ${ }^{59,62}$ HLA locus mismatching was identified as a risk factor for recurrence ${ }^{67}$ in one study but not in others. ${ }^{59-61,64}$ A recent study from the United Kingdom demonstrated that the 5- and 10-year recurrence rates after LT were $6 \%$ and $11 \%$, respectively, in their cohort consisting of 69 patients with $\mathrm{AIH}$, in which $87 \%$ of patients were under longterm maintenance treatment with corticosteroids after LT. ${ }^{58}$ Compared to the recurrence rate of $27 \%$ in their previous report in 1999 in patients without long-term corticosteroid therapy, ${ }^{59}$ the authors concluded that long-term corticosteroid use in combination with immunosuppressive agents was associated with a lower frequency of recurrence.

In general, progressing to cirrhosis and graft failure requiring re-transplantation is uncommon, even when $\mathrm{AIH}$ recurs in the graft. ${ }^{65}$ However, the mechanisms that cause recurrent $\mathrm{AIH}$ after LT remain unclear. Furthermore, there are also substantial differences between adults and pediatric patients with de novo $\mathrm{AIH}$, which substantiates the need for more precise diagnostic guidelines in this area. ${ }^{68,69}$ When recurrence occurs in the graft, the strength of immunosuppression should be reinforced with re-administration or dosing-up of corticosteroids, or the addition of other immunosuppressive agents.

Table 2. Incidence and Risk Factors of Recurrence of AIH after LT

\begin{tabular}{|c|c|c|c|c|c|}
\hline Center sites & Time period & Year & No. & Incidence & Time to recurrence, yr \\
\hline Madrid, Spain ${ }^{62}$ & 1988-1996 & 1998 & 27 & $9(33)$ & $2.6 \pm 1.5$ \\
\hline Birmingham, UK ${ }^{59}$ & NA & 1999 & 47 & $13(28)$ & $2.4(0.5-5.3)$ \\
\hline Paris, France ${ }^{63}$ & 1985-1992 & 1999 & 15 & $3(20)$ & $1.6(1-2.5)$ \\
\hline New York, US ${ }^{64}$ & 1988-1995 & 2000 & 24 & $6(25)$ & $1.3 \pm 0.2$ \\
\hline Boston, $\mathrm{US}^{54}$ & 1983-1998 & 2000 & 12 & $5(42)$ & NA \\
\hline Rochester, US ${ }^{57}$ & 1985-1998 & 2001 & 41 & $7(17)$ & $4.6 \pm 1$ \\
\hline Dallas, US ${ }^{60}$ & 1984-1998 & 2002 & 55 & $11(20)$ & NA \\
\hline Paris, France ${ }^{56}$ & 1985-1992 & 2003 & 17 & $7(41)$ & $2.5 \pm 1.7$ \\
\hline Colorado, US $^{55}$ & 1988-2006 & 2008 & 66 & $23(34.8)$ & 4.3 \\
\hline Alberta, Canada ${ }^{61}$ & NA & 2009 & 46 & $11(24)$ & $4 \pm 1.3$ \\
\hline Birmingham, UK ${ }^{58}$ & $1999-2014$ & 2016 & 69 & $5(7)$ & $3.8(1.5-7.3)$ \\
\hline
\end{tabular}

Data are presented as number (\%), mean $\pm \mathrm{SD}$, or median (range).

AIH, autoimmune hepatitis; LT, liver transplantation; NA, not available.

Table 3. Characteristics of Acute-Onset AIH

Approximately 25\%-46\% of AIH

A continuous spectrum from "genuine" acute hepatitis to acute exacerbation of chronic hepatitis

Frequently ANA seronegative and/or normal IgG

Liver histology is crucial for diagnosis; centrilobular necrosis, lobular and perivenular necroinflammatory activity

Corticosteroids as effective as in classic $\mathrm{AIH}$

AIH, autoimmune hepatitis; ANA, anti-nuclear antibodies; IgG, immunoglobulin G. 


\section{SPECIAL PRESENTATIONS: ACUTE-ONSET AIH}

AIH could develop as an acute form, severe hepatitis, or acute liver failure (ALF) (Table 3). The definition of acute-onset AIH has not yet been strictly determined, and therefore the prevalence of acute-onset AIH varies depending on the definition and cohort. The EASL Clinical Practice Guidelines state that approximately 25\% of AIH patients present with an acute-onset;, ${ }^{5,70}$ in a Korean cross-sectional study $46.4 \%$ of all AIH cases were acuteonset, defined as the presence of recent-onset symptoms $(\leq 30$ days). ${ }^{10}$ Another recent Italian cohort demonstrated that $43 \%$ of AIH patients met the criteria of acute-onset, defined as $>10$ $\times$ ULN of transaminases and $>5 \mathrm{mg} / \mathrm{mL}$ of bilirubin. ${ }^{71}$ On the other hand, the frequency of acute hepatitis was almost $11 \%$ in a Japanese nation-wide, cross-sectional study, when histological diagnosis was employed for the diagnosis of acute hepatitis. ${ }^{9}$ It is of note that this lower number indicated the frequency of histologically determined "genuine" acute hepatitis, but did not include acute exacerbation of chronic hepatitis. Acute-onset AIH may contain two different clinical entities; acute exacerbation of chronic $\mathrm{AIH}$ and genuine acute-onset $\mathrm{AIH}$ without any chronicity. But liver histology is dynamically changing during disease process and it could be extremely challenging to differentiate these two conditions even with an experienced pathologist.

It is important to note that acute-onset $\mathrm{AIH}$ frequently lacks the typical serological findings of $\mathrm{AIH}$, such as positive autoantibodies or elevated serum IgG. A Japanese case series involving 86 patients with acute-onset AIH disclosed that 27\% patients with acute-onset AIH were ANA negative $(<1: 40)$, and there was no elevation of serum IgG in more than 50\% of cases. ${ }^{72}$ As a result, when encountering $\mathrm{AIH}$ patients with elevated transaminases but who lack serological diagnostic markers, the possibility of AIH is not commonly considered by physicians. This can easily lead to a delayed diagnosis and treatment, and progression to severe hepatitis or ALF; therefore, a liver biopsy is crucial for making a diagnosis of acute-onset AIH. Unlike classic and chronic $\mathrm{AIH}$, centrilobular necrosis rather than portal inflammation appears to be characteristic of acute-onset AIH. ${ }^{73-75}$ However, a recent histological study of acute-onset AIH demonstrated that this type of AIH could represent the entire spectrum of liver histology from acute hepatitis to chronic hepatitis with various activity and levels of fibrosis, and that there were no pathognomonic features for acute-onset $\mathrm{AIH}^{76}$ According to this study, several histological findings, including lobular and perivenular necroinflammatory activity, pigmented macrophages, and a cobblestone appearance of hepatocytes, in addition to those present in classic AIH may be beneficial for pathological diagnosis.

Regarding the treatment of acute-onset $\mathrm{AIH}$, corticosteroids appear to be similarly effective as in classic $\mathrm{AIH}$, without significant adverse effects. ${ }^{71,72,75,77,78}$ The prompt initiation of high- dose intravenous (i.v.) corticosteroids (1 g methylprednisolone for 3 consecutive days followed by i.v. $1 \mathrm{mg} / \mathrm{kg} /$ day prednisolone or i.v. $1.5 \mathrm{mg} / \mathrm{kg} /$ day prednisolone) appears to be safe and may prevent progression to liver failure in patients with acutesevere $\mathrm{AIH}^{79}$ A recent retrospective study from India suggested that use of corticosteroids for acute-on-chronic liver failure (ACLF) with AIH as an acute insult was significantly associated with a shorter intensive care unit stay and improvement in the 90-day survival rate, while the incidence of sepsis was comparable to patients that were not treated with corticosteroids. ${ }^{80}$ The revised Asian Pacific Association for the Study of the Liver consensus recommendations for ACLF indicate that "diagnosis of ACLF-AIH requires liver biopsy (transjugular route preferred)," and "corticosteroid therapy should be considered for a select group of patients presenting with ACLF-AIH." ${ }^{\text {11 }}$ LT is a single option for very severe cases who cannot be saved with medical treatment alone, but the optimal timing of LT is difficult to determine. $^{82}$

\section{FUTURE PERSPECTIVE}

Whereas the long-term outcome of patients with $\mathrm{AIH}$ is excellent and comparable to those in the general population, several unmet needs still remain to be addressed. First, early diagnosis of the disease and treatment intervention with immunosuppressive agents should be standardized globally, especially in Asia-Pacific regions where viral hepatitis is endemic and $\mathrm{AIH}$ is believed to be rare. As described, more than $70 \%$ of patients with $\mathrm{AIH}$ were diagnosed after progression to cirrhosis in South Asia, and 20\% to 25\% in Korea and the United Kingdom. Furthermore, a cross-sectional study in the United Kingdom demonstrated that the current treatment regimen is not uniform (29 treatment regimens were reported among 1,249 patients in secondary care units), and as a result, the remission rates were just 59\%. ${ }^{83}$ Therefore, it is crucial to widely disseminate clinical practice guidelines for the management of $\mathrm{AIH}$, making it possible to diagnose the disease at an earlier stage and to properly manage with a standardized treatment policy.

Second, the health-related quality of life (HrQOL), another important endpoint of treatment, should be carefully considered. Several studies indicated that the HrQOL of AIH patients was severely impaired and that depression appears to be a dominan symptom affecting well-being, despite clinical and biochemica features also being present. ${ }^{84-86}$ In particular, the use of corticosteroids are significantly associated with a decreased HrQOL, ${ }^{85,86}$ and the use of budesonide appears to improve the HrQOL. ${ }^{84}$ These observations highlight the unmet need for an alternative treatment regimen without corticosteroids, not only for improving the long-term outcome in those who exhibit incomplete responses or who are intolerant to corticosteroids, but also for patients who demonstrate an excellent response but who also require long-term maintenance therapy. 


\section{CONFLICTS OF INTEREST}

No potential conflict of interest relevant to this article was reported.

\section{ORCID}

Atsushi Tanaka

https://orcid.org/0000-0002-6358-5283

\section{REFERENCES}

1. Manns MP, Lohse AW, Vergani D. Autoimmune hepatitis: update 2015. J Hepatol 2015;62(1 Suppl):S100-S111.

2. Floreani A, Restrepo-Jiménez P, Secchi MF, et al. Etiopathogenesis of autoimmune hepatitis. J Autoimmun 2018;95:133-143.

3. Alvarez F, Berg PA, Bianchi FB, et al. International Autoimmune Hepatitis Group report: review of criteria for diagnosis of autoimmune hepatitis. J Hepatol 1999;31:929-938.

4. Hennes EM, Zeniya M, Czaja AJ, et al. Simplified criteria for the diagnosis of autoimmune hepatitis. Hepatology 2008;48:169-176.

5. European Association for the Study of the Liver. EASL Clinical Practice Guidelines: autoimmune hepatitis. J Hepatol 2015;63:9711004.

6. Wang Z, Sheng L, Yang Y, et al. The management of autoimmune hepatitis patients with decompensated cirrhosis: real-world experience and a comprehensive review. Clin Rev Allergy Immunol 2017;52:424-435.

7. Yoshizawa K, Matsumoto A, Ichijo T, et al. Long-term outcome of Japanese patients with type 1 autoimmune hepatitis. Hepatology 2012;56:668-676.

8. Kim BH, Choi HY, Ki M, Kim KA, Jang ES, Jeong SH. Populationbased prevalence, incidence, and disease burden of autoimmune hepatitis in South Korea. PLoS One 2017;12:e182391.

9. Takahashi A, Arinaga-Hino T, Ohira H, et al. Autoimmune hepatitis in Japan: trends in a nationwide survey. J Gastroenterol 2017;52:631-640.

10. Kim BH, Kim YJ, Jeong SH, et al. Clinical features of autoimmune hepatitis and comparison of two diagnostic criteria in Korea: a nationwide, multicenter study. J Gastroenterol Hepatol 2013;28:128134.

11. Danielsson Borssén Å, Marschall HU, Bergquist A, et al. Epidemiology and causes of death in a Swedish cohort of patients with autoimmune hepatitis. Scand J Gastroenterol 2017;52:1022-1028.

12. Delgado JS, Vodonos A, Malnick S, et al. Autoimmune hepatitis in southern Israel: a 15-year multicenter study. J Dig Dis 2013;14:611-618.

13. Grønbæk L, Vilstrup H, Jepsen P. Autoimmune hepatitis in Denmark: incidence, prevalence, prognosis, and causes of death: a nationwide registry-based cohort study. J Hepatol 2014;60:612617.

14. Haider AS, Kaye G, Thomson A. Autoimmune hepatitis in a demographically isolated area of Australia. Intern Med J 2010;40:281-285.
15. Ngu JH, Bechly K, Chapman BA, et al. Population-based epidemiology study of autoimmune hepatitis: a disease of older women? J Gastroenterol Hepatol 2010;25:1681-1686.

16. Puustinen L, Barner-Rasmussen N, Pukkala E, Färkkilä M. Incidence, prevalence, and causes of death of patients with autoimmune hepatitis: a nationwide register-based cohort study in Finland. Dig Liver Dis 2019;51:1294-1299.

17. van Gerven NM, Verwer BJ, Witte BI, et al. Epidemiology and clinical characteristics of autoimmune hepatitis in the Netherlands. Scand J Gastroenterol 2014;49:1245-1254.

18. Werner M, Prytz H, Ohlsson B, et al. Epidemiology and the initial presentation of autoimmune hepatitis in Sweden: a nationwide study. Scand J Gastroenterol 2008;43:1232-1240.

19. Tanaka A, Mori M, Matsumoto K, Ohira H, Tazuma S, Takikawa $\mathrm{H}$. Increase trend in the prevalence and male-to-female ratio of primary biliary cholangitis, autoimmune hepatitis, and primary sclerosing cholangitis in Japan. Hepatol Res 2019;49:881-889.

20. Koay LB, Lin CY, Tsai SL, et al. Type 1 autoimmune hepatitis in Taiwan: diagnosis using the revised criteria of the International Autoimmune Hepatitis Group. Dig Dis Sci 2006;51:1978-1984.

21. Grønbæk L, Vilstrup H, Pedersen L, Christensen K, Jepsen P. Family occurrence of autoimmune hepatitis: a Danish nationwide registry-based cohort study. J Hepatol 2018;69:873-877.

22. de Boer YS, van Gerven NM, Zwiers A, et al. Genome-wide association study identifies variants associated with autoimmune hepatitis type 1. Gastroenterology 2014;147:443-452.

23. Gurung A, Assis DN, McCarty TR, Mitchell KA, Boyer JL, Jain D. Histologic features of autoimmune hepatitis: a critical appraisal. Hum Pathol 2018;82:51-60.

24. Zeng T, Yu J, Tan L, et al. Noninvasive indices for monitoring disease course in Chinese patients with autoimmune hepatitis. Clin Chim Acta 2018;486:135-141.

25. Migita K, Horai Y, Kozuru H, et al. Serum cytokine profiles and Mac-2 binding protein glycosylation isomer (M2BPGi) level in patients with autoimmune hepatitis. Medicine (Baltimore) 2018;97:e13450.

26. Wang J, Malik N, Yin M, et al. Magnetic resonance elastography is accurate in detecting advanced fibrosis in autoimmune hepatitis. World J Gastroenterol 2017;23:859-868.

27. Hartl J, Denzer U, Ehlken H, et al. Transient elastography in autoimmune hepatitis: timing determines the impact of inflammation and fibrosis. J Hepatol 2016;65:769-775.

28. Hartl J, Ehlken H, Sebode M, et al. Usefulness of biochemical remission and transient elastography in monitoring disease course in autoimmune hepatitis. J Hepatol 2018;68:754-763.

29. Wu S, Yang Z, Zhou J, et al. Systematic review: diagnostic accuracy of non-invasive tests for staging liver fibrosis in autoimmune hepatitis. Hepatol Int 2019;13:91-101.

30. Johnson PJ, McFarlane IG. Meeting report: International Autoimmune Hepatitis Group. Hepatology 1993;18:998-1005.

31. Muratori P, Granito A, Pappas G, Muratori L. Validation of simplified diagnostic criteria for autoimmune hepatitis in Italian patients. 
Hepatology 2009;49:1782-1783.

32. Yeoman AD, Westbrook RH, Al-Chalabi T, et al. Diagnostic value and utility of the simplified International Autoimmune Hepatitis Group (IAIHG) criteria in acute and chronic liver disease. Hepatology 2009;50:538-545.

33. Arcos-Machancoses JV, Molera Busoms C, Julio Tatis E, Bovo MV, Martín de Carpi J. Accuracy of the simplified criteria for autoimmune hepatitis in children: systematic review and decision analysis. J Clin Exp Hepatol 2019;9:147-155.

34. Tanaka A, Ma X, Yokosuka O, et al. Autoimmune liver diseases in the Asia-Pacific region: proceedings of APASL symposium on $\mathrm{AIH}$ and PBC 2016. Hepatol Int 2016;10:909-915.

35. Gordon V, Adhikary R, Appleby V, et al. Diagnosis, presentation and initial severity of autoimmune hepatitis (AIH) in patients attending 28 hospitals in the UK. Liver Int 2018;38:1686-1695.

36. van den Brand FF, van der Veen KS, de Boer YS, et al. Increased mortality among patients with vs without cirrhosis and autoimmune hepatitis. Clin Gastroenterol Hepatol 2019;17:940947.

37. Borssén ÅD, Palmqvist R, Kechagias S, et al. Histological improvement of liver fibrosis in well-treated patients with autoimmune hepatitis: a cohort study. Medicine (Baltimore) 2017;96:e7708.

38. Choi J, Choi GH, Lee D, et al. Long-term clinical outcomes in patients with autoimmune hepatitis according to treatment response in Asian country. Liver Int 2019;39:985-994.

39. Lamers MM, van Oijen MG, Pronk M, Drenth JP. Treatment options for autoimmune hepatitis: a systematic review of randomized controlled trials. J Hepatol 2010;53:191-198.

40. Manns MP, Czaja AJ, Gorham JD, et al. Diagnosis and management of autoimmune hepatitis. Hepatology 2010;51:21932213.

41. Pape S, Gevers TJG, Belias M, et al. Predniso(lo)ne dosage and chance of remission in patients with autoimmune hepatitis. Clin Gastroenterol Hepatol 2019;17:2068-2075.

42. Manns MP, Woynarowski M, Kreisel W, et al. Budesonide induces remission more effectively than prednisone in a controlled trial of patients with autoimmune hepatitis. Gastroenterology 2010;139:1198-1206.

43. Lohse AW, Gil H. Reactivation of autoimmune hepatitis during budesonide monotherapy, and response to standard treatment. J Hepatol 2011;54:837-839.

44. Peiseler M, Liebscher T, Sebode M, et al. Efficacy and limitations of budesonide as a second-line treatment for patients with autoimmune hepatitis. Clin Gastroenterol Hepatol 2018;16:260267.

45. Liberal R, de Boer YS, Andrade RJ, et al. Expert clinical management of autoimmune hepatitis in the real world. Aliment Pharmacol Ther 2017;45:723-732.

46. Sievers TM, Rossi SJ, Ghobrial RM, et al. Mycophenolate mofetil. Pharmacotherapy 1997;17:1178-1197.

47. Efe C, Hagström H, Ytting $H$, et al. Efficacy and safety of mycophenolate mofetil and tacrolimus as second-line therapy for patients with autoimmune hepatitis. Clin Gastroenterol Hepatol 2017;15:1950-1956.

48. De Lemos-Bonotto M, Valle-Tovo C, Costabeber AM, Mattos AA, Azeredo-da-Silva ALF. A systematic review and meta-analysis of second-line immunosuppressants for autoimmune hepatitis treatment. Eur J Gastroenterol Hepatol 2018;30:212-216.

49. Santiago P, Schwartz I, Tamariz L, Levy C. Systematic review with meta-analysis: mycophenolate mofetil as a second-line therapy for autoimmune hepatitis. Aliment Pharmacol Ther 2019;49:830 839.

50. Dörner T, Posch MG, Li Y, et al. Treatment of primary Sjögren's syndrome with ianalumab (VAY736) targeting B cells by BAFF receptor blockade coupled with enhanced, antibody-dependent cellular cytotoxicity. Ann Rheum Dis 2019;78:641-647.

51. Adam R, Karam V, Delvart V, et al. Evolution of indications and results of liver transplantation in Europe: a report from the European Liver Transplant Registry (ELTR). J Hepatol 2012;57:675688.

52. Singal AK, Guturu P, Hmoud B, Kuo YF, Salameh H, Wiesner RH. Evolving frequency and outcomes of liver transplantation based on etiology of liver disease. Transplantation 2013;95:755-760.

53. The Japanese Liver Transplantation Society. Liver transplantation in Japan: registry by the Japanese Liver Transplantation Society. Japanese J Transplant 2015;52:134-147.

54. Ayata G, Gordon FD, Lewis WD, et al. Liver transplantation for autoimmune hepatitis: a long-term pathologic study. Hepatology 2000;32:185-192.

55. Campsen J, Zimmerman MA, Trotter JF, et al. Liver transplantation for autoimmune hepatitis and the success of aggressive corticosteroid withdrawal. Liver Transpl 2008;14:1281-1286.

56. Duclos-Vallée JC, Sebagh M, Rifai K, et al. A 10 year follow up study of patients transplanted for autoimmune hepatitis: histological recurrence precedes clinical and biochemical recurrence. Gut 2003;52:893-897.

57. González-Koch A, Czaja AJ, Carpenter HA, et al. Recurrent autoimmune hepatitis after orthotopic liver transplantation. Liver Transpl 2001;7:302-310.

58. Krishnamoorthy TL, Miezynska-Kurtycz J, Hodson J, et al. Longterm corticosteroid use after liver transplantation for autoimmune hepatitis is safe and associated with a lower incidence of recurrent disease. Liver Transpl 2016;22:34-41.

59. Milkiewicz P, Hubscher SG, Skiba G, Hathaway M, Elias E. Recurrence of autoimmune hepatitis after liver transplantation. Transplantation 1999;68:253-256.

60. Molmenti EP, Netto GJ, Murray NG, et al. Incidence and recurrence of autoimmune/alloimmune hepatitis in liver transplant recipients. Liver Transpl 2002;8:519-526.

61. Montano-Loza AJ, Mason AL, Ma M, Bastiampillai RJ, Bain VG, Tandon P. Risk factors for recurrence of autoimmune hepatitis after liver transplantation. Liver Transpl 2009;15:1254-1261.

62. Prados E, Cuervas-Mons V, de la Mata M, et al. Outcome of 
autoimmune hepatitis after liver transplantation. Transplantation 1998;66:1645-1650.

63. Ratziu V, Samuel D, Sebagh M, et al. Long-term follow-up after liver transplantation for autoimmune hepatitis: evidence of recurrence of primary disease. J Hepatol 1999;30:131-141.

64. Reich DJ, Fiel I, Guarrera JV, et al. Liver transplantation for autoimmune hepatitis. Hepatology 2000 0ct;32(4 Pt 1):693-700.

65. Montano-Loza AJ, Bhanji RA, Wasilenko S, Mason AL. Systematic review: recurrent autoimmune liver diseases after liver transplantation. Aliment Pharmacol Ther 2017;45:485-500.

66. Visseren T, Darwish Murad S. Recurrence of primary sclerosing cholangitis, primary biliary cholangitis and auto-immune hepatitis after liver transplantation. Best Pract Res Clin Gastroenterol 2017;31:187-198.

67. Balan V, Ruppert K, Demetris AJ, et al. Long-term outcome of human leukocyte antigen mismatching in liver transplantation: results of the National Institute of Diabetes and Digestive and Kidney Diseases Liver Transplantation Database. Hepatology 2008;48:878-888.

68. Kerkar N, Vergani D. De novo autoimmune hepatitis: is this different in adults compared to children? J Autoimmun 2018;95:26-33.

69. Kerkar N, Yanni G. 'De novo' and 'recurrent' autoimmune hepatitis after liver transplantation: a comprehensive review. J Autoimmun 2016;66:17-24.

70. Takahashi H, Zeniya M. Acute presentation of autoimmune hepatitis: does it exist? A published work review. Hepatol Res 2011;41:498-504.

71. Muratori P, Carbone M, Stangos G, et al. Clinical and prognostic implications of acute onset of autoimmune hepatitis: an Italian multicenter study. Dig Liver Dis 2018;50:698-702.

72. Joshita S, Yoshizawa K, Umemura T, et al. Clinical features of autoimmune hepatitis with acute presentation: a Japanese nationwide survey. J Gastroenterol 2018;53:1079-1088.

73. Abe K, Kanno Y, Okai K, et al. Centrilobular necrosis in acute presentation of Japanese patients with type 1 autoimmune hepatitis. World J Hepatol 2012;4:262-267.

74. Hofer H, Oesterreicher C, Wrba F, Ferenci P, Penner E. Centrilobular necrosis in autoimmune hepatitis: a histological feature associated with acute clinical presentation. J Clin Pathol 2006;59:246-249.
75. Sonthalia N, Rathi PM, Jain SS, et al. Natural history and treatment outcomes of severe autoimmune hepatitis. J Clin Gastroenterol 2017;51:548-556.

76. Nguyen Canh H, Harada K, Ouchi H, et al. Acute presentation of autoimmune hepatitis: a multicentre study with detailed histological evaluation in a large cohort of patients. J Clin Pathol 2017;70:961-969.

77. Anastasiou OE, Dogan-Cavus B, Kucukoglu 0, et al. Corticosteroid therapy improves the outcome of autoimmune hepatitis-induced acute liver failure. Digestion 2018;98:104-111.

78. Shen Y, Lu C, Men R, Liu J, Ye T, Yang L. Clinical and pathological characteristics of autoimmune hepatitis with acute presentation. Can J Gastroenterol Hepatol 2018;2018:3513206.

79. Zachou K, Arvaniti P, Azariadis K, et al. Prompt initiation of highdose i.v. corticosteroids seems to prevent progression to liver failure in patients with original acute severe autoimmune hepatitis. Hepatol Res 2019;49:96-104.

80. Anand L, Choudhury A, Bihari C, et al. Flare of autoimmune hepatitis causing acute on chronic liver failure: diagnosis and response to corticosteroid therapy. Hepatology 2019;70:587-596.

81. Sarin SK, Choudhury A, Sharma MK, et al. Acute-on-chronic liver failure: consensus recommendations of the Asian Pacific Association for the Study of the Liver (APASL): an update. Hepatol Int 2019;13:353-390.

82. Rahim MN, Liberal R, Miquel R, Heaton ND, Heneghan MA. Acute severe autoimmune hepatitis: corticosteroids or liver transplantation? Liver Transpl 2019;25:946-959.

83. Dyson JK, Wong LL, Bigirumurame T, et al. Inequity of care provision and outcome disparity in autoimmune hepatitis in the United Kingdom. Aliment Pharmacol Ther 2018;48:951-960.

84. Janik MK, Wunsch E, Raszeja-Wyszomirska J, et al. Autoimmune hepatitis exerts a profound, negative effect on health-related quality of life: a prospective, single-centre study. Liver Int 2019;39:215-221.

85. Takahashi A, Moriya K, Ohira H, et al. Health-related quality of life in patients with autoimmune hepatitis: a questionnaire survey. PLoS One 2018;13:e0204772.

86. Wong LL, Fisher HF, Stocken DD, et al. The impact of autoimmune hepatitis and its treatment on health utility. Hepatology 2018;68:1487-1497. 